\title{
THE EFFECTS OF LIGHT-EMITTING DIODES (LED) IRRADIATION TREATMENT ON THE POSTHARVEST PRESERVATION AND MICROBIOLOGICAL QUALITY OF STRAWBERRY (Fragaria ananassa) CV. FESTIVAL
}

\author{
WAN NUR HIDAYAH WAN MOHD NOOR, WAN ZALIHA WAN SEMBOK AND WAN \\ ZAWIAH WAN ABDULLAH*
}

Faculty of Fisheries and Food Science, Universiti Malaysia Terengganu, 21030 Kuala Nerus, Terengganu, Malaysia.

*Corresponding author: wzawiah@umt.edu.my

http://doi.org/10.46754/umtjur.2021.10.015

\begin{abstract}
Strawberry (Fragaria Ananassa sp.) is non-climacteric, aggregate fruit with a rich source of anthocyanin and vitamin C. However, strawberry has short storage life and is very susceptible to decay and physiological deterioration process. Therefore, emitting the post-harvest LED light to strawberries during cold storage has been studied. This work aimed to evaluate different LED light treatment effects to retain post-harvest quality and microbial quality of the strawberry. The post-harvest parameters that have been used are weight loss, colour, firmness, total soluble solids, titratable acidity, and $\mathrm{pH}$. The microbial parameter such as Escherichia coli count, Salmonella count, total plate count, and mold count would also has been assessed. Strawberries were irradiated under three different LED light types (white, blue and red). Blue and red LED lights have different wavelengths, which are $470 \mathrm{~nm}$ and $630 \mathrm{~nm}$. The strawberry fruits were stored at $5^{\circ} \mathrm{C}$ in a cold room and study parameter were assessed in 10 days. There were statistically significant differences $(p<0.05)$ between the treatment of weight loss, firmness, $\mathrm{pH}$, and total soluble solid. The best treatment for this experiment is blue LED light which showed the lowest weight loss, retained the colour and delayed firmness loss of the strawberry. This study also proved that blue LED light could reduce the growth of E.coli. In conclusion, LED light produces minimal heat, which improves food safety and preserves post-harvest quality of strawberry.
\end{abstract}

Keywords: LED, post-harvest quality, microbiological, irradiation treatment, strawberry.

\section{Introduction}

Strawberry (Fragaria Ananassa sp.) is a nonclimacteric aggregate fruit that belongs to the family Rosaceae and genus Fragaria. It is a hybrid cultivar cultivated in temperate regions worldwide for its tasty fruit. Fragaria $x$ ananassa plants have short, woody stems and a basal rosette of compound leaves, with three coarsely toothed leaflets. The plant is suitable to grow in well-drained soil and can grow either in semi-shade or no shade. Strawberry is usually red in colour during ripening but can be green, white, or yellowish depending on the cultivar. Strawberry is a highly commercial fruit and most often consumed fresh. Strawberry is rich in carbohydrates, vitamin $\mathrm{C}$ and natural antioxidants such as flavonoids and phenolic compounds (Erkan \& Wang, 2008). Flavonoids have been demonstrated to have inflammatory, anti-allergenic, anti-aging and anti-carcinogenic activity (Lopez da Silva et al., 2007). Strawberry can be processed into various food products such as ice creams, jams, cakes, preserves, fruit juices, mousses and candies. Nevertheless, strawberry has a short post-harvest life caused by relatively increased metabolic activity and sensitivity towards fungal decay such as grey mold (Botrytis cinera) and Rhizopus. It is also susceptible to mechanical injury and physiological deterioration because of its lack of protective rind and soft texture (Amar et al., 2010). Post-harvest life of strawberry is also short due to physical damage during handling, water loss, physiological disorder and high susceptibility to spoilage microorganisms (Caleb et al., 2016). 
Hanenberg et al. (2016) has reported that the use of LEDs as supplemental overhead lighting improves overall berry fruit quality such as flavour, vitamin $\mathrm{C}$, total soluble solid content (TSS, ${ }^{\circ}$ Brix) and can efficiently increase crop yield and flower production when applied directly to the leaf canopy and fruit within a greenhouse environment.

The treatment of light emitted diode (LED) and cold storage temperature can be applied to prolong strawberry shelf life. It can at least reduce the mold growth, senescence of strawberry, and prolong the strawberry's postharvest life. LEDs have been demonstrated to enrich or preserve the nutritional quality of fruits in post-harvest phase, inactive pathogenic bacteria and reduce fungal infections. This work examined the effect of different LED lights on post-harvest preservation and microbiological quality of strawberry.

\section{Materials and Methods}

\section{Material Preparation}

Strawberry (Fragaria ananassa) fruits of the variety Festival were bought from the Al-Manshoor Farm, located in Cameron Highlands, Pahang Darul Makmur. The amount of strawberry fruit needed in this study was about 300 , with stages two and three of the maturity indexes. The strawberries were transported to the Postharvest Technology Laboratory of Universiti Malaysia Terengganu in the evening in insulated boxes. The strawberries were sorted to eliminate unripe and overripe fruits as well as any samples with apparent defects. Only fruits with a uniform ripening stage, size, colour, shape and without physical injuries were selected. The strawberries were pre-cooled to a temperature of $5^{\circ} \mathrm{C}$. The sample was assessed at days $0,2,4,6,8$ and 10 .

\section{Light Emitted Diode (LED) Light Treatment}

Three types of LED light treatment were used in this study. The treatment included white irradiated (control, Treatment 1), red light irradiated, $630 \mathrm{~nm}$ (Treatment 2), and blue light irradiated, $470 \mathrm{~nm}$ (Treatment 3). This study was conducted in the $5^{\circ} \mathrm{C}$ cool room for 10 days. The analysis was evaluated at every twoday interval.

\section{Post Harvest Quality Evaluation Weight Loss}

The quality of the strawberries was assessed. The strawberries were weighed using a top balance and repeated for three replications. The difference between initial and final fruit weight was considered as total weight loss during each storage interval and calculated as percentages on a fresh-weight basis by the standard method.

Percentage of weight loss (\%) = initial weight of strawberry ( $g$ ) - final weight of strawberry ( $g$ )

initial weight of strawberry $(\mathrm{g}) \times 100$

Weight measured after removing excess water by using tissue paper. Each sample's net weight registered on days $0,2,4,6,8$ and day 10. Three replicate packages were analyzed.

\section{Colour}

The colour value of strawberry (three strawberries per treatment) was measured at the center of the region and recorded with a Minolta Chroma Meter (Model Konica Minolta, CR-400). Colour meter was calibrated using the standard white plate before measurement (Caner \& Aday, 2009). The redness index ( $\left.a^{*}\right)$ of strawberry was determined.

\section{Firmness}

A texture analyzer (Model TA-XT plus) was used to record the textural properties of all samples from each treatment.

A flat-ended cylindrical probe of stainless steel having $10 \mathrm{~mm}$ diameter was used for compression (Jain \& Pathare, 2004). The sample was placed under the probe that moved downwards. During the test run, the sample 
was recorded every $0.02 \mathrm{~s}$ and plotted in a force against a time graph. The observation of force till the bio-yield point of skin hardness with an interval of one second recorded on texture analyzer will be used for fitting the force and time. The experimental error has been determined in terms of percent internal uncertainty for force. Fruit flesh firmness was assessed using a texture analyzer and the reading was recorded in $\mathrm{N}$ force. For each treatment, three strawberries were analyzed at room temperature (Caner \& Demir, 2008).

\section{Total Soluble Solid}

Nutrients are deposited as starch, which during the ripening process is transformed into sugars during the development of flesh of a fruit. The progression of the ripening process leads to increased sugar levels. Measurement of TSS of strawberries (fresh and stored) were done by extracting the juice, and few drops of juice were poured over the designated platform of the refractometer ranges from 0 to $32^{\circ}$ brix and then allowing the light to pass through the prism. This instrument measures the refractive index, which indicates how much a light beam is 'bent' when it passes through the fruit juice. The TSS was directly recorded from the scale and expressed as a percentage (\%) (Caney \& Aday, 2009).

\section{Titratable Acidity and $\mathrm{pH}$}

Titratable acidity was measured by titration method and AOAC (1990) was suggested. Three strawberries for each treatment were cut and smashed into pieces, then squeezed into juice. Five $\mathrm{ml}$ of the juice were dissolved with $45 \mathrm{ml}$ of distilled water to prepare a strawberry aliquot. Five $\mathrm{ml}$ of the aliquot was transferred into a conical flask and with two or three drops of phenolphthalein was added as an indicator. The titration was done with $0.1 \mathrm{M} \mathrm{NaOH}$ until the solution was stable with a $\mathrm{pH}$ of 8.1 or 8.2 . $\mathrm{NaOH}$ volume to neutralize the aliquot was recorded after the colour of the aliquot changes from colourless to pink. The recorded result was calculated as the percentage of acid with the formula:

\section{Microbial Analysis Evaluation}

\section{Escherichia Coli Count}

For this analysis, samples of three strawberries were mixed with $225 \mathrm{ml}$ of saline water and homogenized in a stomacher for one minute. In all cases, serial dilutions from the microbial extracts were prepared in $9 \mathrm{ml}$ sterile water. The dilution was dispensed into Eosine Methylene Blue (EMB) agar and spread on agar's surface. The inoculated plates were incubated at $37^{\circ} \mathrm{C}$ for 24 hours. The agar plate was inspected and typical E.coli colonies were determined.

\section{Statistical Analysis}

Data was subjected to one-way Analysis of Variance (ANOVA) and comparison of means followed by Tukey's tests, using SPSS version 23.

\section{Results and Discussion}

\section{Weight Loss}

Figure 1 shows that weight loss of emitted LED light and non-emitted LED light strawberry increased gradually during the storage period. There were statistically significant difference between treatment $(\mathrm{p}<0.05)$ of total weight loss. On day 10, the control sample has the highest weight loss while blue (Treatment 3) has the least weight loss.

The T1, T2 and T3 treatments achieved eight days of shelf life, with an average loss of $34.8 \%$, $18.8 \%$ and $16.7 \%$ respectively. Interestingly, the weight loss of $\mathrm{T} 1$ (control) was significantly higher than other treatments. The loss of fruit weight during post-harvest storage can be attributed to water loss because fruits contain a large amount of water that is relatively easy to evaporate. T1 treatment shows a high value of weight loss due to the weak protective barrier. Strawberries revealed weight loss due to their soft texture and lack of protective rind (Amar et al., 2010). Deterioration of strawberries was due to loss of weight during the storage period due to high respiration and transpiration rate. Loss of moisture content may be due to 
differences in relative humidity between the storage environment inside and the atmosphere (Abdelgader et al., 2011). The strawberries that have been emitted with LED light could reduce weight loss. It has been reported that emitting strawberries with LED light showed the lowest weight loss and weight loss increased as the storage period increased (Qi et al., 2011). These data indicate that the treatments can effectively prevent strawberry moisture loss by strengthening the skin, the migration of moisture from the inner of fruits to the surface slowed down, leading to reduced moisture loss and possibly reduced respiration rate (Qi et al.,
2011). Water loss involves a difference in the gradient of water vapor pressure between the internal part of the fruit and the environment outside, which causes moisture loss from the fruit by vapor phase diffusion (Maftoonanazad et al., 2005).

Therefore, the irradiation of LED light towards strawberries could be a barrier, restricting the water transfer and protecting fruit skin from mechanical injury. Also, the blue LED had the best protective effect on weight loss, which indicates that it might have a synergistic effect on retarding the weight loss of strawberries.

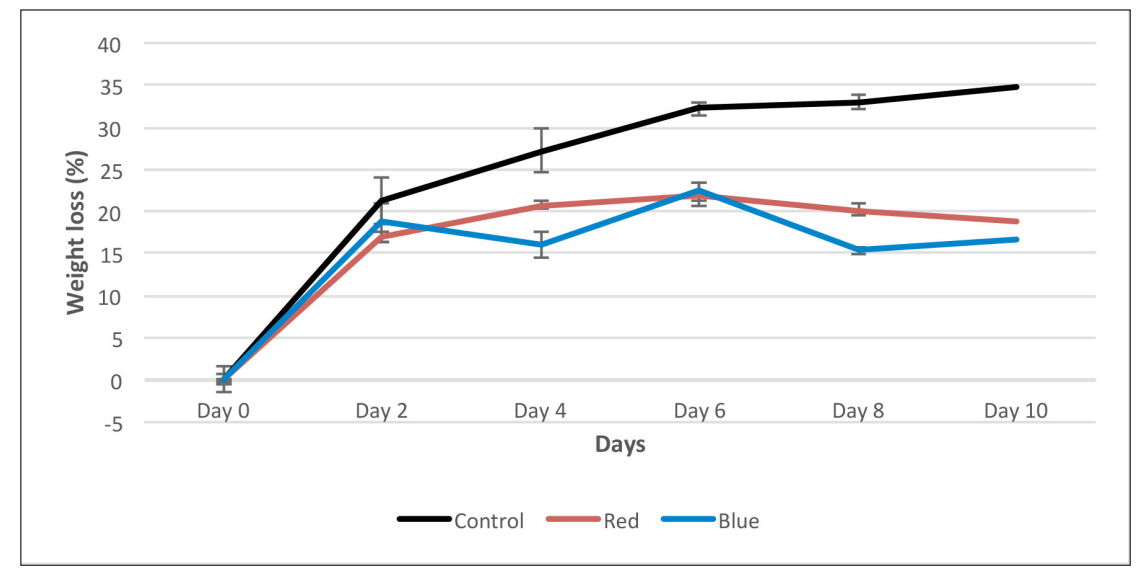

Figure 1: Percentage of weight loss of strawberries emitted with different LED lights

\section{Firmness}

The firmness of strawberries was analyzed using a texture analyzer. Jawadul et al. (2014) stated that the texture and firmness is a critical quality attribute in consumer acceptability of fresh fruit and vegetable. Firmness is one of the essential parameters reflecting post-harvest storage characteristic of fruits. It is related to tissue softening. Loss of firmness caused by the loss of integrity of cell wall structure leads to wilting and shrinking fruit (Qi et al., 2011).

Figure 2 shows the decrease of firmness for all treatments within the storage period. $\mathrm{T} 2$ and $\mathrm{T} 3$ treatments showed a high value of firmness compared to T1 (control). There was a significant difference between the treatments and time of storage $(\mathrm{p}<0.05)$. The graph shows that irradiation of LED light towards strawberry had maintained the firmness of strawberry. 


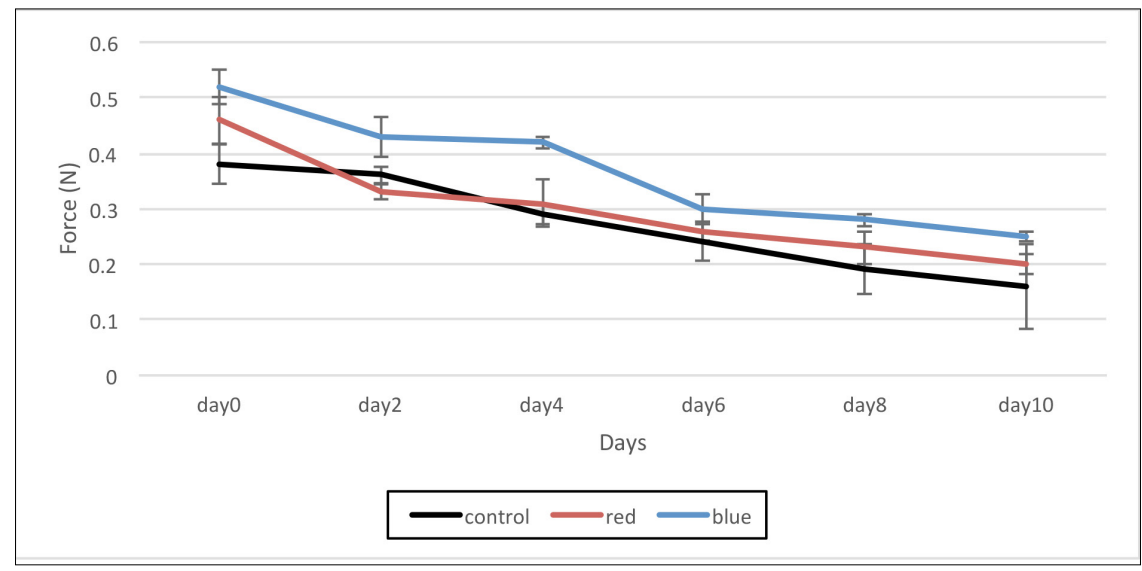

Figure 2: Firmness of strawberries emitted with different LED lights

\section{Colour}

Strawberry is rich in anthocyanin compounds, which refer to their red colour. The ripening process for strawberries has been correlated to the anthocyanin content. Emitting LED light to the fruit may increase anthocyanin accumulation, retard microbe growth and delay the colour change of fruit

From Figure 3, the redness $\left(a^{*}\right)$ of sample strawberry with different LED light treatments showed that there were significant differences $(p<0.05)$ between each treatment. Chromatic a* of strawberry decreased over time irrespective of the treatments. Chromaticity a* value is the measurement of greenness or redness, which is varying from 60 to +60 .

The decrease in $a^{*}$ value of red and blue LED light treatments was slower than white LED light treatment. The lowest redness is shown by white LED light treatment (21.29), while the red LED treatment treated strawberries (24.24) and blue LED light treatment treated strawberries have the greatest chromaticity, a* value (26.19). Similar to the result obtained by Nereide et al. (2012), the chromaticity of $a^{*}$ value of blue LED light treated strawberries differed from the white LED light treated strawberries.

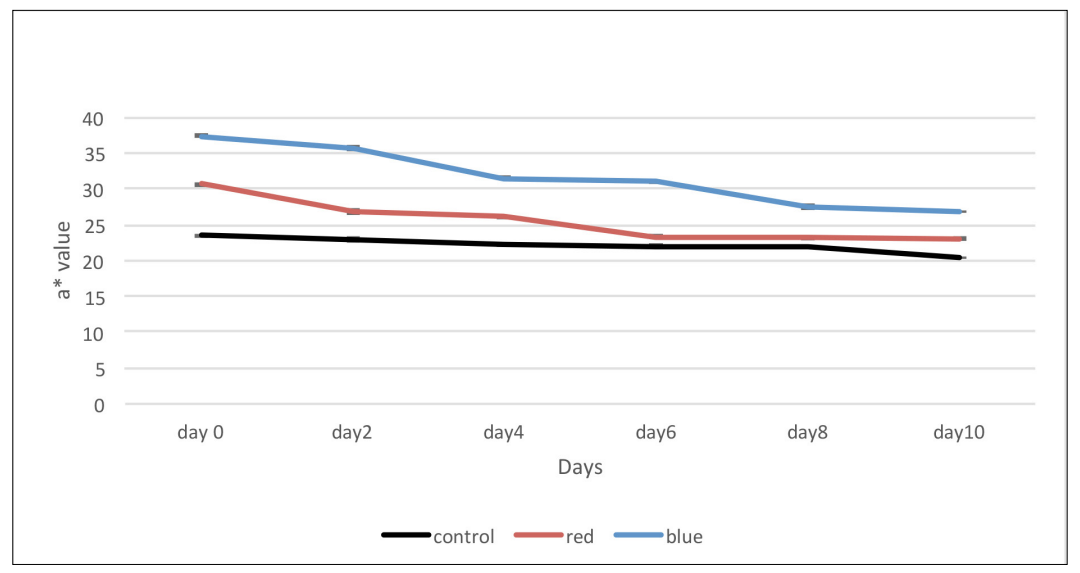

Figure 3: The $\mathrm{a}^{*}$ value for the strawberry sample emitted with different LED lights for 10 days 


\section{Firmness}

Figure 4 shows the decrease of firmness for all treatments within the storage period. T2 and T3 treatments showed a high value of firmness compared to T1. After 10 days of storage, $\mathrm{T} 1$ had the lowest firmness $(0.16 \mathrm{~N})$ while red $(0.2 \mathrm{~N})$ and blue had the right firmness $(0.25 \mathrm{~N})$, respectively. There was a significant difference between the treatments and time of storage $(\mathrm{p}<$ 0.05 ). These results showed that irradiation of LED light towards the strawberries had maintained their firmness. Irradiation treatment of LED light was able to improve the properties in extending the shelf life while maintaining their quality.

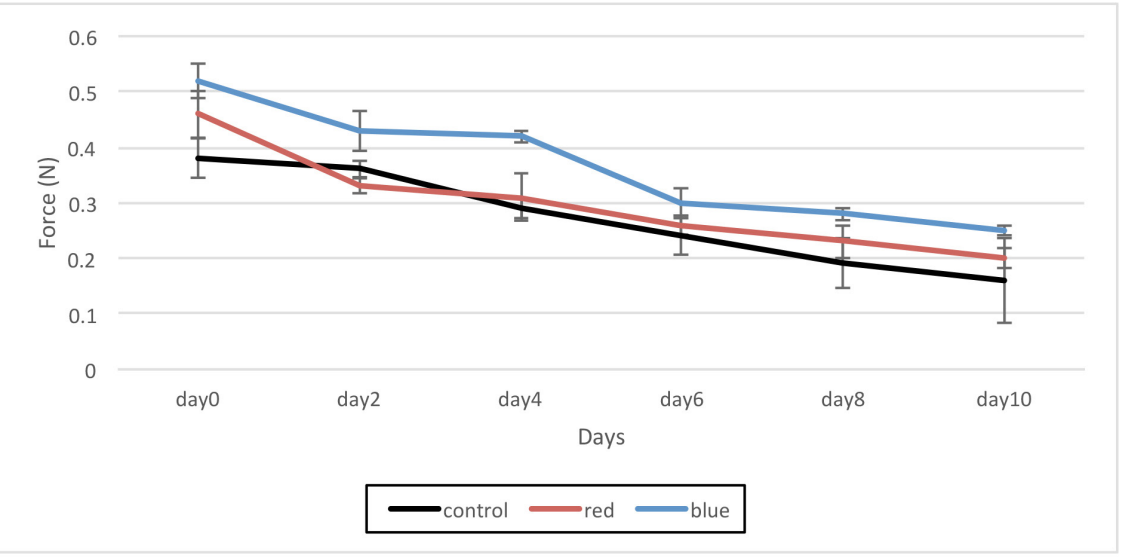

Figure 4: Firmness for a sample of strawberries emitted with different LED lights for 10 days

As the process of fruit ripening progresses, shortening of chain length of pectin substance occurs with an increase in pectinesterase activities (Yaman et al., 2002). The biochemical basis of strawberry softening is not clear. Strawberry softening has been associated with the degradation of the middle lamella of cortical parenchyma cells, resulting in a dramatic increase in pectin solubilization, with slight pectin molecular weight changes small decrease in the content of hemicellulose (Pilar et al., 2008). Strawberry has a soft rind due to high water activity and thin epidermis. The epidermis acts as a protective barrier towards the pathogen. This part is sensitive as it can cause lesions so that the pathogen can penetrate the strawberry, thus leading to food spoilage (Leque et al., 2003). Blue LED light strengthens the epidermis so the penetration of the pathogen towards the strawberry can be reduced. Strawberry contains natural antioxidants and phenols (Caleb et al.,
2016). These are evolutionary antimicrobial components of the fruit that allow it to defend itself from microorganisms by inhibiting its microbiological activities.

\section{Total Soluble Solid}

Figure 5 shows the total soluble solid for a sample of strawberries emitted with different LED lights. The trend of the total soluble solid was decreasing over time. On day 0 , there was no significant difference $(p<0.05)$ between the treatments. The TSS value in white LED light treatment slightly lowered solid soluble content compared to the red LED light treatment and blue LED light treatment where 6.0 brix $^{\circ}$ was observed for white LED light treated strawberries and 6.2 brix $^{\circ}$ for strawberries that emitted with red LED light and blue LED light. A similar result was obtained by Asghari et al. (2009), who claimed that the treatment did not significantly affect the TSS of treated strawberry fruit compared to control. 


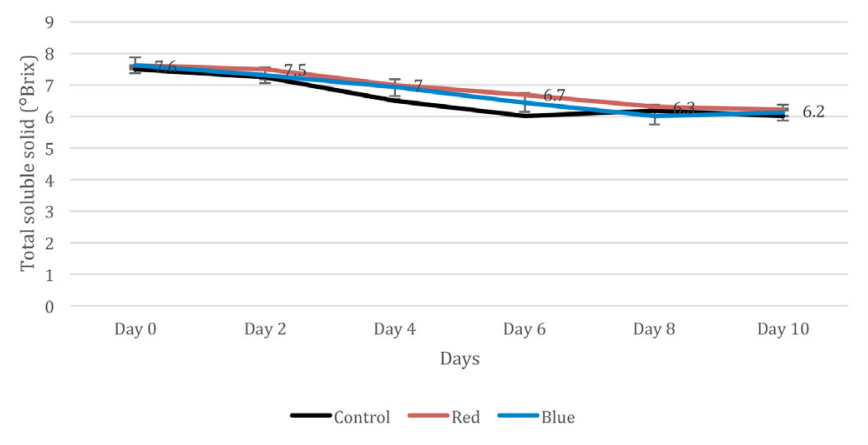

Figure 5: Total soluble solid content for the sample of strawberries emitted with different LED lights

The reduction of TSS might be related to anthocyanin's biosynthesis induced by sugar such as sucrose, glucose, fructose, or galactose (Al-Juhaimi et al., 2013). According to TanadaPalmu and Grosso (2005), an increase in TSS in control strawberries can be expected to increase soluble solid content during ripening and a decrease in mature fruit due to respiration. A plausible explanation for the observed increment in TSS is the considerable water loss suffered by strawberries during storage. Indeed, the more significant change in TSS occurred in those strawberries which suffered the most significant water loss. The solubilization of the cell wall polyuronides and hemicellulose in ripe strawberries might also increase TSS value (Tanada-Plamu \& Grosso, 2005).

\section{Titratable Acidity}

Fresh produce is the most important source of many vitamins in the human diet. Strawberry has higher vitamin $\mathrm{C}$ content, which is suitable for human consumption and the dominant acid is citric acid. Figure 6 shows the titratable acidity for strawberry that has been emitted with different LED lights. Titratable acidity decreased gradually during storage in all treatments, but the decrease in red and blue LED light treatment strawberries was comparatively slower compared to in white LED light treatment. Increased level of acidity in fruits can be caused by increased respiration rate and production of high-level carbon dioxide, $\mathrm{CO} 2$, which affected the glycolytic enzyme systems and resulted in the accumulation of acids (Lester et al., 1999).

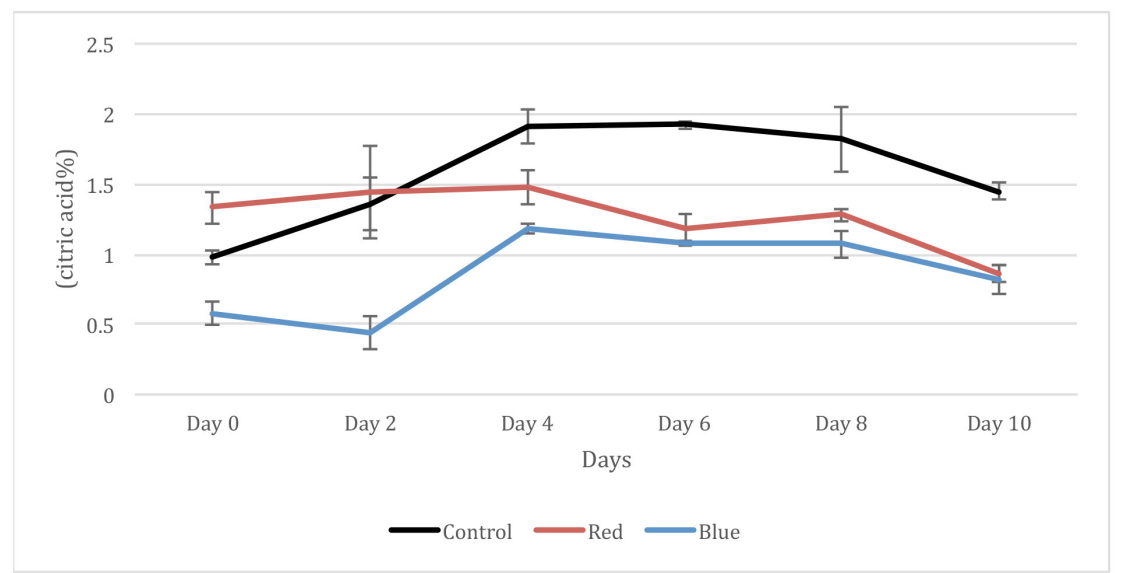

Figure 6: Titratable acidity for a sample of strawberries emitted with different LED lights 
On day 0 , there was a significant difference $(p<0.05)$ between all treatments. The initial titratable acidity of all treatments was $3.5 \%$ and $4.5 \%$ citric acid. On day four, the strawberries emitted by red and blue LED lights had a significantly lower titratable acidity value than white LED light treatment. At the end of the storage period, strawberries emitted with white LED light had the highest titratable acidity values. There was slightly increased titratable acidity on day four and day six caused by a higher respiration rate and high carbon dioxide production. On day 10, white LED light treated strawberry had the highest value of titratable acidity $(1.45 \%)$ while red $(0.85 \%)$ and blue LED light $(0.82 \%)$. These indicate that the irradiation of red and blue LED lights has reduced the ripening process and maintained the titratable acidity of strawberries. The results revealed that the total anthocyanins increased as they depended on Pg3G production, the secondary anthocyanin (Pg3MG) showed a distinct increase at a quantitative level under blue LED light treatment (Kondo et al., 2014). From the data, the blue LED light treated strawberries had the least acidity value as they act as antifungal and antibacterial against the broad spectrum of food spoilage microorganisms. The reduction in titratable acidity value was due to the conversion of acid into sugars and their further utilization in fruit's metabolic process (Ochiki et al., 2014). The acidity of the fruit is a necessary trait to determine the quality of fruit and its acceptability.

\section{pH}

The average fresh strawberry has a low $\mathrm{pH}$ value of about 3.3 to 3.6 which makes the strawberries taste sour. The $\mathrm{pH}$ of strawberries is one of the critical quality attributes in consumer acceptability of fresh fruits and vegetables. Figure 7 shows the $\mathrm{pH}$ value for strawberries that has been emitted with different LED lights. The trend of the $\mathrm{pH}$ value was decreasing over the storage period.

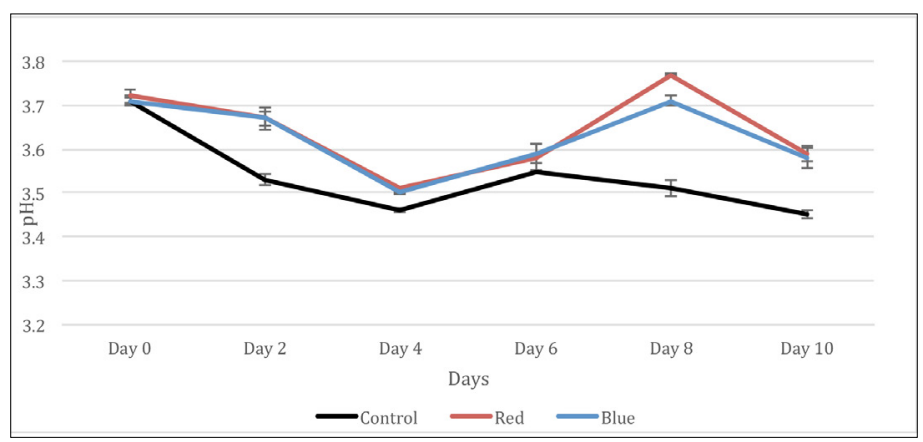

Figure 7: $\mathrm{pH}$ value for the sample of strawberries emitted with different LED light for 10 days

On day 0 , there was a significant difference $(p<0.05)$ between all treatments. The initial $\mathrm{pH}$ value of all treatments was 3.7. On day four, the strawberries emitted by red and blue LED lights have a significantly higher $\mathrm{pH}$ value than than those emitted by white LED light treatment. At the end of the storage period, the strawberries emitted with white LED light had the lowest $\mathrm{pH}$ values. There was a slightly increased $\mathrm{pH}$ value for all treatments on day 6 due to higher respiration rate that accelerated the strawberry's ripening. On day 10, white LED light treated strawberries have the lowest value of $\mathrm{pH}$ (3.45) while red (3.59) and blue LED light (3.60). From these data, blue LED light treated strawberries have the least $\mathrm{pH}$ value as its antifungal and antibacterial can delay the growth of the infected pathogen. In agreement with the previous findings, the tomato also significantly affects when emitting LED light towards fresh produce (Dhakal \& Baek., 2014; Nájera et al., 2018). 


\section{Escherichia Coli Count}

Figure 8 shows the E.coli count $(\log \mathrm{CFU} / \mathrm{mL})$ of the sample strawberries with different LED light treatments. Bacteria were grown dramatically at all samples stored under different irradiation of LED light. The initial microbial count of strawberries was $4.7 \log \mathrm{CFU} / \mathrm{mL}$. During the storage, there was a significant difference $(p<0.05)$ between treatments. After four days of storage, strawberries treated with white LED light had the highest value in E.coli count (5.1 $\log \mathrm{cfu} / \mathrm{mL}$ ). On the last day of storage, blue LED light treated strawberries had the lowest value (5.1 $\log \mathrm{CFU} / \mathrm{mL})$ compared to white (5.5 $\log \mathrm{CFU} / \mathrm{mL}$ ), and red LED light treatments (5.3 $\log \mathrm{CFU} / \mathrm{mL}$ ).
In this study, the trend of $E$. coli growth was significantly different for each emitting LED light. Retardation by the blue LED light for bacteria $E$. coli, aerobic methicillin resistant, Staphylococcus aureus and Pseudomonas aeruginosa (PA) has also been reported (Guffy \& Wilborn, 2006). All the LED light treatments did not exceed the spoilage levels five log CFU/ $\mathrm{ml}$ until 10 days of storage. This result is similar to the result obtained by Lipovsky (2010), showing a significant reduction of the CFUs of the two strains of non-pathogenic $E$. coli on the surface of the fruit compared to the population inside the fruit when emitted with LED light. The visible blue LED light also enhances the cell death in E. coli due to ROS induction (Pal et al., 2009) for 10 days.

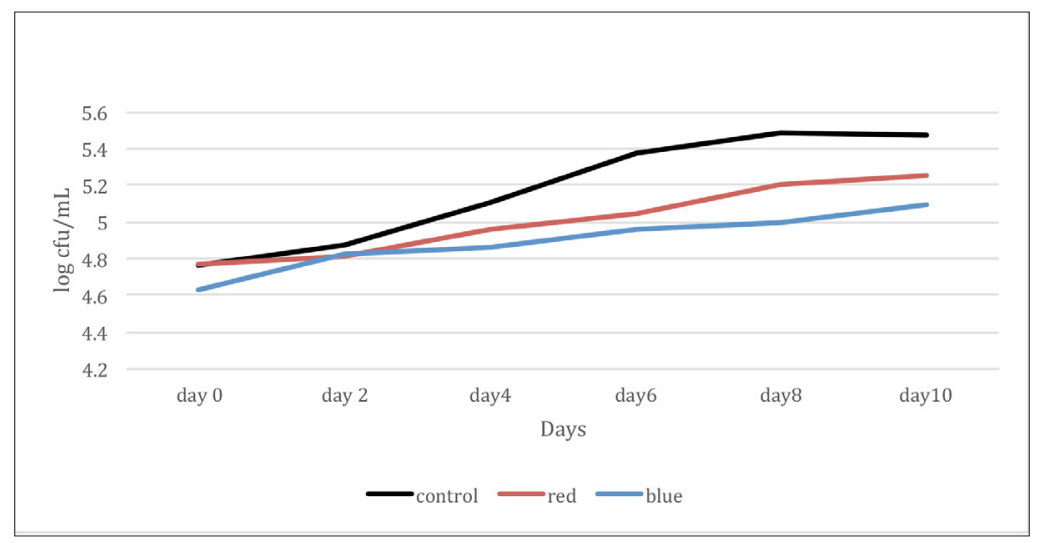

Figure 8: E.coli count for a sample of strawberries emitted with different LED lights

\section{Conclusion}

We have investigated the effect of different irradiation of LED lights on the post-harvest quality of strawberries. The chemical analysis consisting of total soluble solid (TSS), $\mathrm{pH}$ and titratable acidity has been well observed. The physical analysis consists of the percentage of weight loss, firmness and colour changes on the surface of strawberries which can also be the parameter to determine the quality of the strawberries. These parameters are fundamental in determining consumer acceptance of consuming fresh produce. The best treatment to prolong the shelf life and maintain the postharvest quality of strawberry is by emitting the blue LED light towards the strawberry during the storage period. Blue LED light can also inhibit and delay the microbe growth such as E.coli, Salmonella, and mould. Different LEDs combined with chilling temperatures could be applied to preserve strawberry fruits and minimize the risk of foodborne disease.

\section{Acknowledgements}

The authors would like to thank the staff of Postharvest Technology Laboratory and Crop 
Science Laboratory, Faculty of Fisheries and Food Science, Universiti Malaysia Terengganu for their technical assistance.

\section{References}

Abdelgader, M. O., \& Ismail, I. A. (2011). Application of gum Arabic for coating dried mango slices. Pakistan Journal Nutrition, 10(5): 457-462.

Asghari Marjanlo, A., Mostofi Y., Shoeibi S.H., \& Fattahi A. (2009). Effect of cumin essential oil on postharvest decay and some quality factors of strawberry. Journal of Medical Plant, 8: 25-43.

Caleb, O. J., Wegner, G., Rolleczek, C., Herppich, W. B., Geyer, M., \& Mahajan, P. V. (2016). Hot water dipping: Impact on postharvest quality, individual sugars, and bioactive compounds during storage of 'Sonata'strawberry. Scientia Horticulturae, 210, 150-157.

Caner, C., \& Aday, M. S. (2009). Maintaining quality of fresh strawberries through various modified atmosphere packaging. Packaging Technology and Science, 22(2), 115-122.

Caner, C., \& Demir, M. (2008). Extending quality of fresh strawberries by equilibrium modified atmosphere packaging. European Food Research \& Technology, 227, 15751583.

Dhakal, R., \& Baek, K. H. (2014). Metabolic alternation in the accumulation of free amino acids and $\gamma$-aminobutyric acid in postharvest mature green tomatoes following irradiation with blue light. Horticulture, Environment and Biotechnology, 55(1), 36-41.

Erkan, M., Wang, S. Y., \& Wang, C. Y. (2008). Effect of UV treatment on antioxidant capacity, antioxidant enzyme activity and decay in strawberry fruit. Postharvest Biology and Technology, 48, 163-171.
Guffy, J.S., \& Willborn, J. (2006). In vitro bactericidal effect of $405 \mathrm{~nm}$ and $470 \mathrm{~nm}$ blue light. Photomed and Laser Surgery, 24, 684-68.

Lester, G.E., \& Grusak, M.A. (1999). Postharvest application of calcium and magnesium to honeydew and netted muskmelons. Journal of the American Society of Holticultural Science, 124, 545-552.

Leque, J., Fauconnier, M-L., Chammai, A., Bronner, R., \& Blee, E. (2003). Formation of plant cuticle: evidence for the occurrence of the peroxygenase pathway. Plant Journal, 36. Pg.155-164.

Lipovsky, A., Nitzen, Y., Gedanken, A., \& Lubart, R. (2010). Visible light induced killing of bacteria as a function of wavelength. Lasers in Surgery and Medicine, 42, 467-472.

Maftoonanazad, N., \& Ramaswary, H.S. (2005). Postharvest shelf-life extension of avocado. LWT-Food Science and Technology, 38(6): 617-624.

Nájera, C., Guil-Guerrero, J. L., Enríquez, L. J., Álvaro, J. E., \& Urrestarazu, M. (2018). LED-enhanced dietary and organoleptic qualities in postharvest tomato fruit. Postharvest Biology and Technology, 145, 151-156.

Pal, S., Tak, Y.K., Joarder, J., Kim, W., Lee, J.E., Han, M.S., \& Song, J.M. (2009). Nanocrystalline silver supported on activated carbon matrix from hydrosol: antibacterial mechanism under prolonged incubation temperatures. Journal of Nanoscience and Nanotechnology, 9. pp 2092- 2103.

Pilar Hernandez- Munoz, Eva A., Valerie D.V., Dinoraz V., \& Rafael G. (2008). Effect of chitosan coating combined with postharvest calcium treatment on strawberry. Food Chemistry, 110: 428-435. 
Qi, H., Hu, W., Jiang, A., Tian, M., \& Li, Y. (2011). Extending shelf-life of fresh cut 'Fuji' apples with chitosan coating. Innovative Food Science and Emerging Technologies, 12, 62-66.

Tanada-Palmu, P. S., \& Grosso, C.R.F. (2005). Effect of edible wheat-gluten based film and coating on refrigerated strawberry (Fragaria ananassa) quality. Postharvest Biology Technology, 36:199-208.

Yaman, O., \& L. Bayoindrli. (2002). Effect of an edible coating and cold storage on shelflife and quality of cherries. LebensmittelWissenschaft Und -Technologie, 35: pp46150 . 
